\title{
Five Year Study of a Unique, Eco-friendly, Menstrual Cup, That Enhances the Quality of Women's Lives During Menstruation
}

\author{
Alfred Adib Shihata ${ }^{1,}$, , Steven Brody ${ }^{2}$ \\ ${ }^{1}$ Department of Medicine and Science, Scripps Research Institution, San Diego, USA \\ ${ }^{2}$ School of Medicine, University of California, San Diego, USA
}

Email address:

alfred@femcap.com (A. A. Shihata),sab12@cox.net (S. Brody)

${ }^{*}$ Corresponding author

To cite this article:

Alfred Adib Shihata, Steven Brody. Five Year Study of a Unique, Eco-friendly, Menstrual Cup, That Enhances the Quality of Women's Lives During Menstruation. Journal of Gynecology and Obstetrics. Vol. 7, No. 6, 2019, pp. 161-165. doi: 10.11648/j.jgo.20190706.12

Received: September 11, 2019; Accepted: September 29, 2019; Published: October 23, 2019

\begin{abstract}
Menstrual cups are gaining more popularity among women, [1]. who prefer cups over pads and tampons. The innovative FemmyCycle (Figure 2) has several advantages over traditional menstrual cups, which has led to its increased popularity [2]. The objective of this study is to mitigate and correct the possible or potential side effects caused by traditional menstrual cups (Figure 3). The most common side effect among traditional cups is leakage. "Despite the fact that 1.9 billion women globally are of menstruating age - spending on average 65 days a year dealing with menstrual blood flow, few good quality studies exist that compare sanitary products", says senior author Penelope Phillips-Howard, a public health epidemiologist from the Liverpool School of Tropical Medicine in the UK. [3]. We focused our study on all causes of leakage and other side effects caused by traditional menstrual cups. Methods of corrective and preventive actions included; the no-spill design, (Figure $4 \&$ Figure 5), use of a removal ring instead of a rigid stem, the use of a flask shaped body rather than the conical shape of traditional menstrual cups, (Figure 2 \& Figure 3), the elimination of suction holes and the need to manually open the cup. We analyzed 834 independent reviews by women who have had prior experience with traditional menstrual cups to obtain objective results. The implementation of these design changes and enhancing the written and video instructions produced measurable, objective, positive results in favor of the FemmyCycle. We analyzed all these reviews for FemmyCycle to measure the effects on leakage and other side effects. We selected the reviewers who volunteered to compare the FemmyCycle with their experience with traditional cone shaped menstrual cups. There were many reasons why women preferred FemmyCycle, but the most common by far was the prevention of leakage followed by the ease of insertion, ease of removal and comfort when inserted. The implementation of the design changes from a conical shaped cup with a removal stem to a flask shape cup with a removal ring, in addition to the added no-spill feature and enhanced instructional material, improved the acceptability of the FemmyCycle.
\end{abstract}

Keywords: Menstrual Cup, FemmyCycle No-spill Design, Pads and Tampons

\section{Introduction}

Women have always tolerated the inconvenience of managing their menstruation. Despite the great advance in gynecology, there are great unmet menstrual hygiene needs among low-income women [4]. Demand for menstrual hygiene products has been neglected, particularly in developing countries, due to lack of resources [5]. In the western world, feminine sanitary products are a necessity of life. On average a woman disposes of 360-400 contaminated pads, tampons and plastic applicators per year [Figure 6]. Figure 6 shows the yearly environmental impact of pads and tampons used by one woman versus the yearly use of one FemmyCycle menstrual cup, and uses between 11,00016,000 pads and tampons in her lifetime (Figure 1). Each year, western women dispose of more than 20 billion 
contaminated pads, tampons, and plastic applicators, which then pollute the environment. In developing countries, women still resort to reused rags, which may be contaminated. Millions of women and girls in developing countries miss up to 65 days of school or work per year, due to the lack of private facilities and access to affordable menstrual sanitary products. They also lack a clean water supply [6]. Missing these days of school or work cause a major barrier to full participation in society. A menstrual cup can be a good solution for menstrual hygiene management in economically challenged settings. Objective evidence through cup color change suggests schoolgirls in rural Africa can use menstrual cups [7].

The Menstrual cup was invented in 1932 however, it was not well accepted by women at that time. Most menstrual cups used today have almost the same exact design of the original cup invented 87 years ago. The Tampon was invented in 1940 which was followed by the strapless pad in 1971. There have been no noticeable advances in menstrual hygiene products until the FemmyCycle was introduced to the Market in 2013 [8].
To both fill the unmet need for a safe, environmentally friendly menstrual product and to improve the quality of life for women during menstruation, Dr. Alfred Shihata the first author of this study developed a unique, reusable menstrual collection device. This device was approved by the United States Food and Drug Administration (FDA) on November 15,2012 , which was soon followed by the approval of the European Union and Health Canada [8].

Tampons are made of a blend of cotton and rayon which is then bleached to achieve a clean, white sterile look. While the United States Environmental Protection Agency (USEPA) banned the harmful bleaching process in 1998, current elemental 'chlorine-free' bleaching process utilize chlorine dioxide which still produces a small number of dioxins. Chlorine dioxide can be toxic even in small quantities.

Despite these notable flaws, tampons became the most popular menstrual product in the western world. Tampons cause vaginal dryness, expose the vagina to chemicals they contain in their production, and increase the risk of Toxic Shock Syndrome [9].

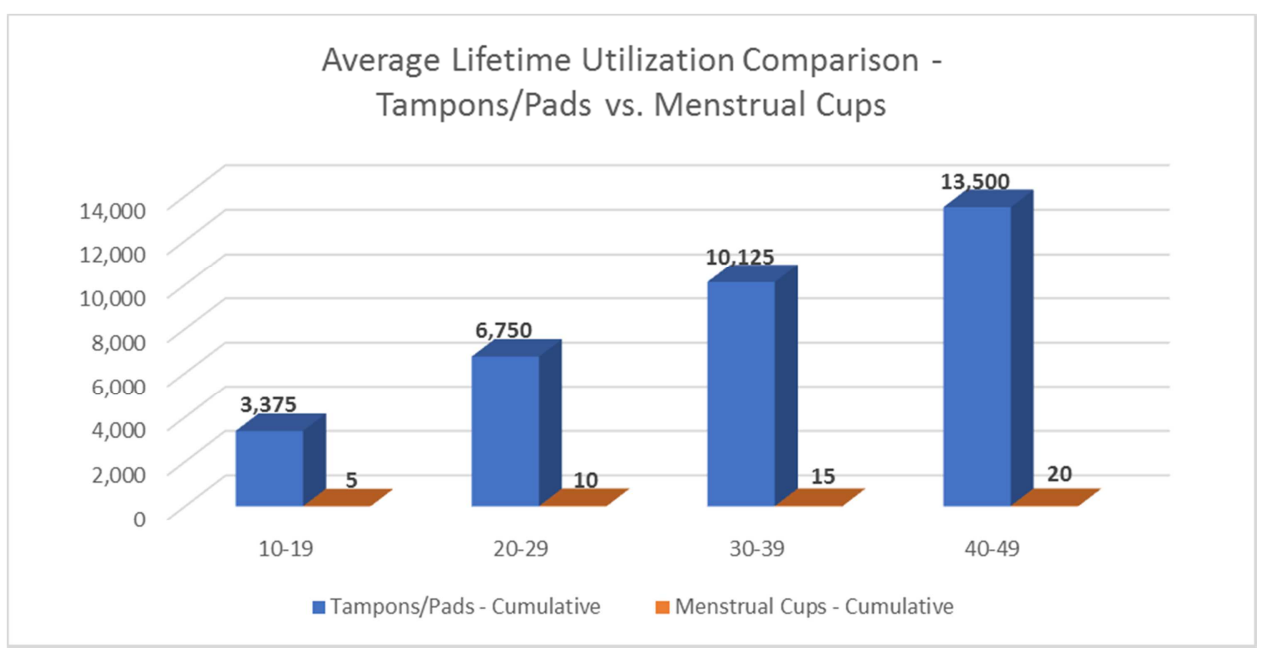

Figure 1. Average Lifetime Utilization Comparison - Tampons/Pads vs. FemmyCycle Menstrual Cups.

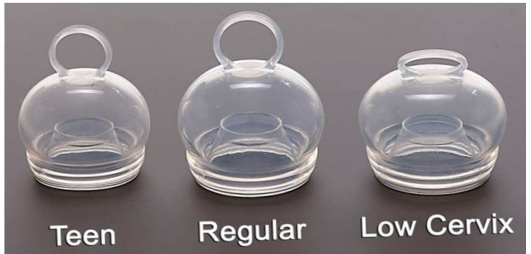

Figure 2. Three sizes FemmyCycle Menstrual cup.

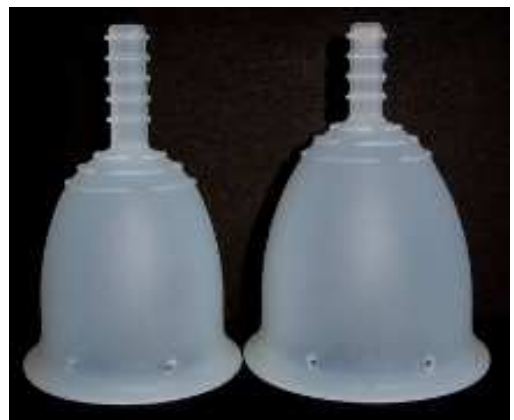

Figure 3. Two sizes Traditional Menstrual cup.

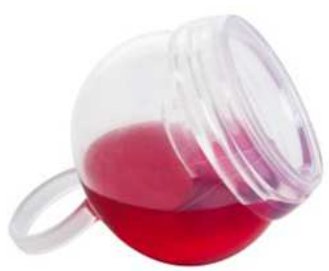

Lid Closed

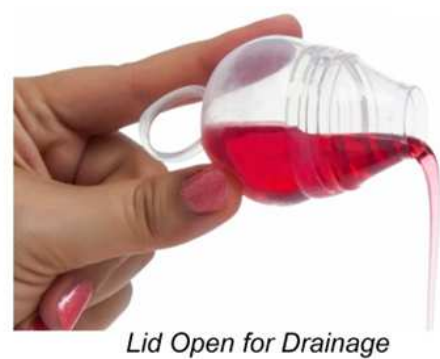

Figure 4. No Spill Design.
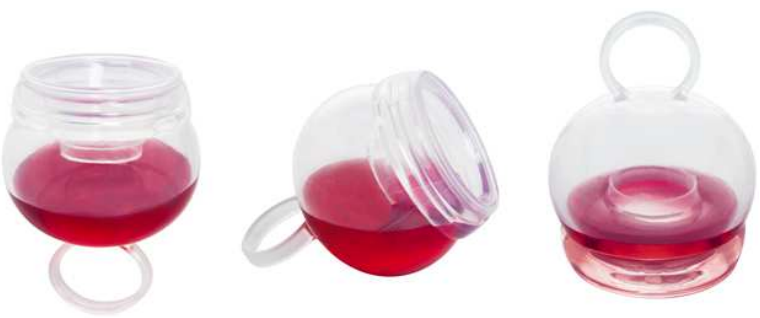

Figure 5. No Spill Design 2. 
Environmental impact of pads and tampons vs. the FemmyCycle $₫$ per year

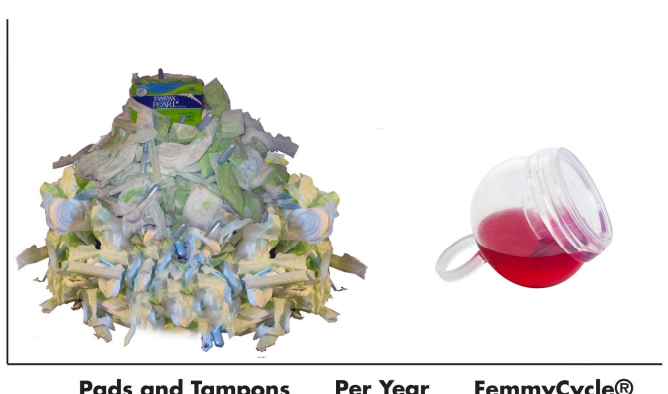

Figure 6. Environmental Impact.

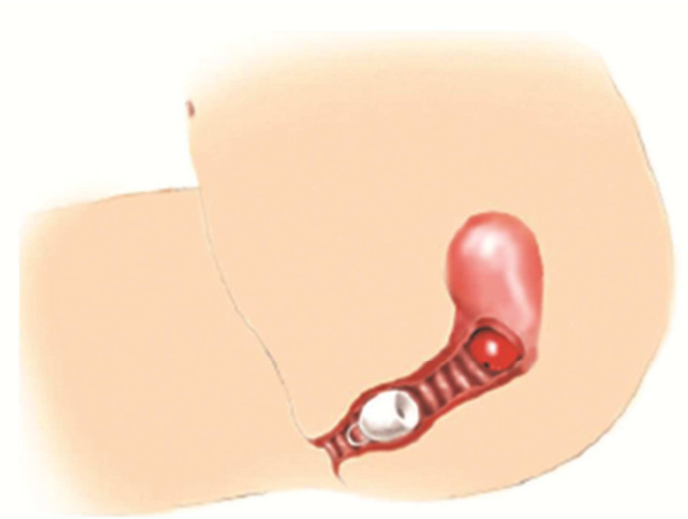

Figure 7. Proper placement.

\section{Materials and Methods}

\subsection{Sizing Details}

The FemmyCycle menstrual cup comes in three sizes to accommodate for variables in women's anatomy and physiology.

The regular size (Figure 2) would be most suitable for adult women who have an average or longer-than-average distance between the vaginal opening and the cervix. The Regular Size FemmyCycle is $5 \mathrm{~cm}$ long and has a diameter of $4.5 \mathrm{~cm}$. The FemmyCycle has the capacity to collect and hold one ounce of menstrual flow and can be reused for up to two years. The device has a removal ring that makes removal possible with a single finger. The device also has resilience and memory that restores the cup to its original shape no matter how many times it has been compressed.

The Low-Cervix Model would be suitable for women who delivered vaginally and / or their cervixes have descended into the vaginal canal. Users of the Low-Cervix cup have a distance between the vaginal opening and the cervix that is shorter than average but not less than 2 inches.

The Teen Size is $10 \%$ smaller in length and diameter than the Regular Size to accommodate younger bleeders and users with a smaller body build.

\subsubsection{Description of the Device}

The FemmyCycle is wineglass-shaped and is made of compressible, medical-grade silicone. The device has a unique funnel lid that directs the blood into the receptacle and prevents liquid from spilling out during removal. The nospill design (Figure 4 \& Figure 5) incorporates a lid meant for containing fluid when closed and pouring fluid out easily and with no mess when opened (Figure 4).

\subsubsection{Cleaning the Device}

Medical-grade silicone can be easily cleaned with mild soap and water.

\subsubsection{Usage}

To collect the menstrual fluid properly, the FemmyCycle should be placed below the cervix into the lower part of the vagina (Figure 7).

\subsection{The Device's Safety and the Silicone's Biocompatibility to the Vaginal Tissues Were Both Evaluated Before the FDA Granted Their Approval of the FemmyCycle}

Enhanced illustrated written, instructions are provided with the device and several video instructions are available online.

Since the FemmyCycle device is sold over the counter, a detailed well illustrated and written step-by-step instruction is provided with each device. The instructions explain the dynamics of the cervix in relation to the vagina clearly. The written and the video instructions explain to women that the axis of the cervix is rarely in a straight line with the vagina, and most frequently the cervix is tilted in any direction. The cervix may be tilted forward and the uterus tilted backwards (also known as a Retroverted uterus), the cervix may be tilted backwards and the uterus forward (known as an Anteverted Uterus) or the cervix may be tilted sideways. The cervix moves upwards during ovulation and downwards during menstruation. For proper collection of the menstrual fluid, the menstrual cup must be placed below the cervix (Figure 7). Women are clearly precautioned that they should never use a menstrual cup if they have any stage of cervical prolapse or if the distance between the vaginal opening and the cervix is less than 2 inches during menstruation. It has a capacity to collect and hold one ounce of menstrual flow and can be reused for two years. The device has a removal ring that facilitates its removal by a single finger.

\subsection{Data Collections}

Eight hundred and thirty-four (834) independent reviews of women who have had prior experience with traditional menstrual cups, were collected and analyzed.

\section{Results}

Eight hundred and thirty-four (834) women (86\%) preferred the FemmyCycle due to the lack of leakage, 92\% reported comfort when compared to the discomfort of the stem poking them of the traditional menstrual cups. Ninety- 
eight (98\%) of users reported that they cannot feel the device at all while it is in placed correctly. Fifty-six, 56\% reported ease of insertion and $82 \%$ reported ease of removal compared to the rigid stem, other traditional menstrual cups [10-14].

Table 1. Comparison between FemmyCycle and Traditional Menstrual Cups.

\begin{tabular}{|c|c|c|}
\hline & FemmyCycle & Traditional Menstrual Cups \\
\hline Leakage & $\begin{array}{l}\text { Very rare and usually occurs when the user has the } \\
\text { wrong size or has placed the device incorrectly. } \\
\text { Designed not to open completely to create suction to } \\
\text { draw fluid into the cup }\end{array}$ & $\begin{array}{l}\text { Very common and usually women are instructed to open rim } \\
\text { manually, women are instructed to insert their fingers deep into the } \\
\text { vagina running her fingers all-round the rim. }\end{array}$ \\
\hline Design & Flask-shape & Cone-shape \\
\hline Rim & Soft and resilient rim & Firm or rigid rim \\
\hline Removal & Flexible ring & Rigid stem \\
\hline Comfort & Cannot feel the cup at all & $\begin{array}{l}\text { The firm rim can press on the urethra causing difficulty during } \\
\text { urination and the stem can poke on the labia. The manufacturer } \\
\text { recommends cutting the stem, which makes the device more difficult } \\
\text { to remove. }\end{array}$ \\
\hline Odor & Less odor & Still have odor \\
\hline
\end{tabular}

\section{Discussion}

Our main objective is to develop a menstrual cup that is designed based on the anatomy and physiology of the cervix and the vagina regardless of age. The traditional menstrual cup comes in two sizes based on age, small size before age 30 and a larger size for women over 30 years old. The FemmyCycle comes in 3 sizes: Regular, Low Cervix and Petite. Instead of a cone shape that is pointed towards the vaginal opening and is easily expelled if the woman coughs or sneezes, the FemmyCycle is designed flask-shaped where the widest diameter is towards the opening of the vagina. This design eliminates involuntary expulsion.

The FemmyCycle is designed with a soft ergonomically designed ring to be removed by a single finger whereas the traditional menstrual cup has a rigid stem, which is awkward to remove and can poke a woman's labia. Ironically the manufactures of traditional cups instruct women to cut the stem, which will make removal more difficult and messier.

Our second objective is to raise awareness about women's basic unmet needs as well as eliminate the stigma and misconception of menstruation [15]. Women prefer menstrual cups over pads and tampons, despite the leakage and other side effects of traditional menstrual cups. The lack of innovation in menstrual hygiene products and disregard for woman's essential needs, inspired the invention of the FemmyCycle. We studied and analyzed 834 independent reviews to learn what women need. That study showed that women need a leak-free, easy to use menstrual cup that protects them for at least 8-12 hours. Nurses, teachers, athletes and military personnel have a great need for such a device.

\section{Conclusion}

Menstrual cups are a welcomed advance for modern active women. The FemmyCycle made a major impact in menstrual hygiene products. The FemmyCycle provides 12 hours of uninterrupted protection that is safer for women and the environment. The FemmyCycle does enhance the quality of women's lives during menstruation, particularly working and athletic women.

This enhancement is attributed to the longer duration of protection. It may also prevent women and girls in developing countries from missing school or work during their menstruation. The FemmyCycle may protect the environment from the yearly disposal of billions of pads, tampons and plastic applicators. This FemmyCycle can help fill a major void in menstrual hygiene products and women's reproductive health worldwide.

\section{Acknowledgements}

We thank the women who volunteered to write a review about their experience with the FemmyCycle in comparison with other menstrual hygiene products, including traditional menstrual cups.

We also thank Ms. Monica Chinisaz, Ms. Megan Steiner, and Julia Barrett-Mitchell, who edited this manuscript. No funding was requested or received from any institution. The authors are solely responsible for the content of this article.

\section{References}

[1] Menstrual Cup Wikipedia has 50 updated references on menstrual cups.

[2] Shihata A, Brody S: An innovative, Reusable Menstrual Cup that enhances the Quality of Women's life during Menstruation. British Journal of Medicine \& Medical Research 4 (19): 3581, 2014.

[3] Penelope A Phillips-Howard, et al. Menstrual cup use, leakage, acceptability, safety, and availability: a systematic review and meta-analysis Lancet Public Health 2019; 4: e37693.

[4] Kuhlmann AS; Peters B. Unmet Menstrual Hygiene Needs Among Low-Income Women: Obstetrics \& Gynecology. 133 (2): 238-244, FEBRUARY 2019. 
[5] Ten VTA. Menstrual hygiene: A neglected condition for the achievement of several millennium development goals. Europe External Policy Advisors. Oct 2007.

[6] Sommer M. Where the education system and women's bodies collide: The social and health impact of girls' experiences of menstruation and schooling in Tanzania. J Adolesc. 2010; 33: 521-529. [PubMed].

[7] Anna Maria van Eijk, Kayla F. Laserson, Elizabeth Nyothach, et al Research, Use of menstrual cups among school girls: longitudinal observations nested in a randomized controlled feasibility study in rural western Kenya, Reproductive Health volume 15, Article number: 139 (2018) Reproductive Health volume 15, Article number: 139 (2018).

[8] https://www.accessdata.fda.gov/cdrh_docs/pdf12/K121857.pd f K121857

[9] Bright R. Dwyer D. Retained menstrual tampons: Hazards and epidemiology. International Society of Technology Assessment in Health Care Meeting; Canmore, Alberta, Canada. 2003.
[10] Liswood R. Internal menstrual protection. Use of a safe and sanitary menstrual cup. Obstet Gynecol. 1959; 13: 539-543. [PubMed].

[11] Wysocki S. New options in menstrual protection. A guide for nurse practitioners. Adv Nurse Pract. 1997; 5: 51-54. [PubMed].

[12] Stewart K. Powell M. Greer R. An alternative to conventional sanitary protection: Would women use a menstrual cup? J Obstet Gynecol. 2009; 29: 49-52. [PubMed].

[13] Pena E. Menstrual protection. Advantages of the menstrual cup. Obstet Gynecol. 1962; 19: 684-714. [PubMed].

[14] Karnaky K. Internal menstrual protection with the rubber menstrual cup. Obstet Gynecol. 1962; 19: 688-691. [PubMed].

[15] Houppert K. The curse-Confronting the last unmentionable taboo: Menstruation. New York: Farrar Straus and Giraux; 1999. 